\title{
Fully automated psychiatric diagnosis: Some new possibilities
}

\author{
MARTIN MORF, PHILIP ALEXANDER, and TOM FUERTH \\ University of Windsor, Windsor, Ontario N9B 3P4, Canada
}

\begin{abstract}
A fully automated psychiatric diagnostic system is outlined that would rely on picturepreference items rather than verbal ones and that would involve a tailored approach rather than conventional "brute-strength" testing. The conditions under which such a system can diagnose patients in a way that might arouse their interest and cooperation are discussed. These include a set of diagnostic categories (perhaps a subset from DSM-III), availability of a single-frame video disk unit allowing random access to each frame, a microprocessor system with a memory capacity of about 32,000 bytes, known base rates of the population of interest, and known probabilities of a particular response to the items for each diagnostic category. Some futuristic extensions of the proposed system are suggested.
\end{abstract}

In this age of microprocessors, it is not surprising that computers play an increasingly important role in personality assessment and in the diagnosis of mental disorders. The focus here will be on automated assessment systems based on self-report data. A mere 10 years ago, use of the computer in assessment based on self-report data was confined to bulk scoring and interpretation, either at a local multiuser computer installation or by means of commercial services (e.g., Finney, 1966; Fowler, 1969; Paitich, 1973). A more recent development has been the introduction of on-line testing that provides an immediate report to the psychologist or psychiatrist (e.g., Johnson, Giannetti, \& Williams, 1978; Johnson \& Williams, 1978). Still more recently, systems that are both on-line and interactive have been developed. These raise the possibility of testing tailored to the individual based on either the fixed-branching or the variablebranching approach (e.g., Bremser \& Davidson, 1978).

A second development of some relevance to diagnosis is a certain shift in interest from words to images. Some say that the old linear, sequential, "left-hemisphere" society is making way for a holistic and instantaneous one more attuned to the "right hemisphere." Speculation aside, our experience has been that many psychiatric inpatients we tested recently were not able to handle the items of the Minnesota Multiphasic Personality Inventory (MMPI). Tailored testing reduces the problem by reducing the number of items that must be answered in most cases, but the recent development of the Picture Preference Test (PPT; Cowan, Auld, \& Begin, 1974) suggests that verbal self-report items can be avoided completely in some situations and to a great extent in others. Each PPT item consists of two simple drawings differing in one or several carefully selected aspects,

Requests for reprints should be sent to Martin Morf, Department of Psychology, University of Windsor, Windsor, Ontario N9B 3P4, Canada. and the subjects' task is to indicate which picture they prefer.

\section{DESIGN OF THE ESPECIALLY RAPID DIAGNOSTIC SYSTEM (ERDS)}

Let us assume that we have decided to develop a system for psychiatric diagnosis that administers picturepreference items using a tailored testing procedure. Somewhat optimistically, we will call it the Especially Rapid Diagnostic System (ERDS). What sort of problems can we expect in the development of ERDS? What useful hardware is available? What would be the specific nature of the diagnostic items?

\section{The Two Assumptions of the Bayesian Model}

The particular method of tailored testing might be a Bayesian procedure like that outlined by Morf and Krane (1973). This approach rests on the two assumptions that there is a set of categories that are exhaustive and mutually exclusive and that the diagnostic items are conditionally independent.

Several years ago, it seemed to many that the best way to obtain a set of categories was to establish them empirically by means of cluster or factor analysis (e.g., Morf, Syrotuik, \& Krznaric, 1977), but the publication of the American Psychiatric Association's (1980) Diagnostic and Statistical Manual of Mental Disorders (DSM-III) casts a somewhat different light on this issue. For purposes of this paper, we will assume that we can find a suitable set of categories in DSM-III, a set that might include second-order categories like "dissociative disorder" and "conduct disorder" to reduce the number of diagnostic groups to a manageable number of about 20.

The second assumption of the Bayesian model, that the items are conditionally independent, means that, given a diagnostic category, knowledge of one item response should not change the probability of another. 
More formally, it means that the following condition should be met: $P\left(a_{j} / c_{i}\right)=P\left(a_{j} / c_{i}, a_{k}\right)$, where $a_{j}$ and $a_{k}$ are the responses a to any two different items $\mathrm{j}$ and $\mathrm{k}$.

This assumption raises two problems. First, in practice, an evaluation of the extent to which it holds true is difficult. The very first difficulty one encounters is that the number of subjects falling into each category is likely to be small (e.g., Gustafson, 1969). Second, most prescriptions offered for coping with items that are not conditionally independent exact a price. For example, one could reduce the number of predictors or diagnostic items and lose information, or one could modify the formulas underlying the procedure and increase immensely the computional effort required. Two apparently promising methods involve selecting predictors in order of redundancy and arranging the predictors in "conditionally independent complexes" (Gustafson, 1969).

\section{Picture-Preference Items}

Given a set of diagnostic categories and a derivation sample of patients whose diagnoses are known, the diagnostic picture-preference items can be developed. Two potential examples are shown in Figure 1. Both belong to a set of items constructed by Rudzinski (1979) to assess thought disorder. The point biserial correlation of Item 75 , obtained for a sample of 70 psychiatric patients, with a composite score of Overall and Gorham's (1962) Brief Psychiatric Rating Scale was .37 , and that of Item 128 was .42 . In the context of Bayesian identification, the item properties of greatest interest are the conditional probabilities $P\left(a_{j} / c_{i}\right)$ of a response a to Item $j$, given membership of the respondent in Category i. For example, if the difference between $P\left(a_{j} / c_{1}\right)$ and $P\left(a_{j} / c_{2}\right)$ is marked, Item $j$ is useful in discriminating between Categories 1 and 2 .

\section{Audiovisual Equipment}

Items like Rudzinski's (1979) are not easily produced by computer graphics. The picture information is difficult to code, the resolution level of the pictures produced tends to be low, and it takes a few seconds to produce the picture on most terminals. Also, the picture is typically produced in a sequential fashion that might be interesting or puzzling to the patient, but that in either case produces an extraneous factor that could affect the response made. The apparently most suitable audio-visual device available is a single-frame video disk unit (EFS-1A, Arvin/Echo Corporation ${ }^{1}$ ). This device reads from a video disk that can store 200 frames on 200 tracks on each of its two sides. A control unit (RC-100, Arvin/Echo Corporation) permits random and instant access to any of the 200 frames on one side of the disk. One version of the control unit, of particular interest here, permits the tracks to be addressed by a digital computer.

\section{The Microcomputer System}

In order to explain the role of the computer in this system, it may be useful to recapitulate some aspects
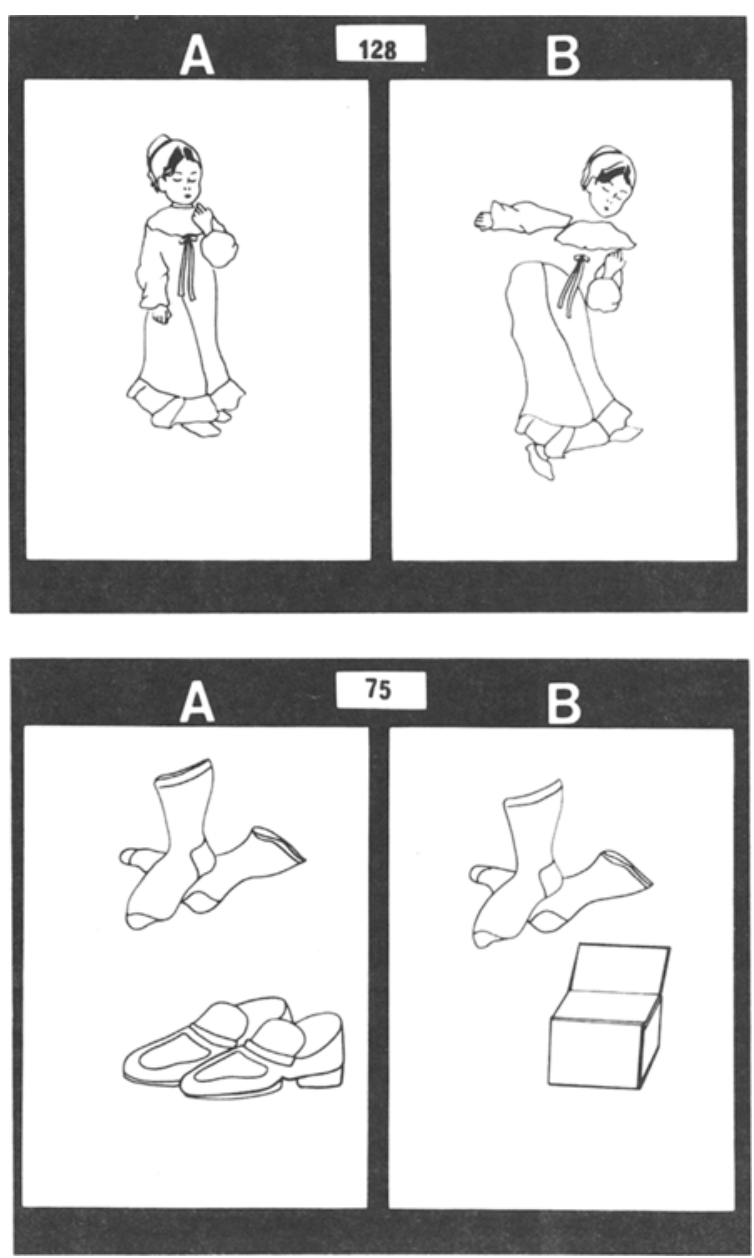

Figure 1. Sample picture-preference items. Item 75 reflects the functional association socks-shoes vs. the clang association socks-box, and Item 128 reflects normal vs. fragmented image. (Copyright 1979 D. Rudzinski. Reprinted by permission.)

of the Bayesian diagnostic procedure described by Morf and Krane (1973). It consists of a series of steps. In the course of each step, a new item is selected and presented. In the present context, the patient indicates a preference for Picture A or B by pressing the appropriate keys on a keyboard. The instructions to the patient include something like: "Make a choice even if you are not sure that you really prefer one picture to the other."

Figure 2 presents a diagram of a simple version of ERDS designed to handle one patient at a time. In particular, it shows the elements that compose the microcomputer system. In some kind of permanent storage, the computer must store the following information: (1) The program that executes the diagnostic procedure. The object code storage requirement for one program used to simulate sequential Bayesian identification on an IBM 360 system required about 16,000 bytes. More efficient programming and program chaining could reduce that amount considerably. (2) The initial probabilities $P\left(c_{i}\right)$ of membership in each diagnostic category $i$. 


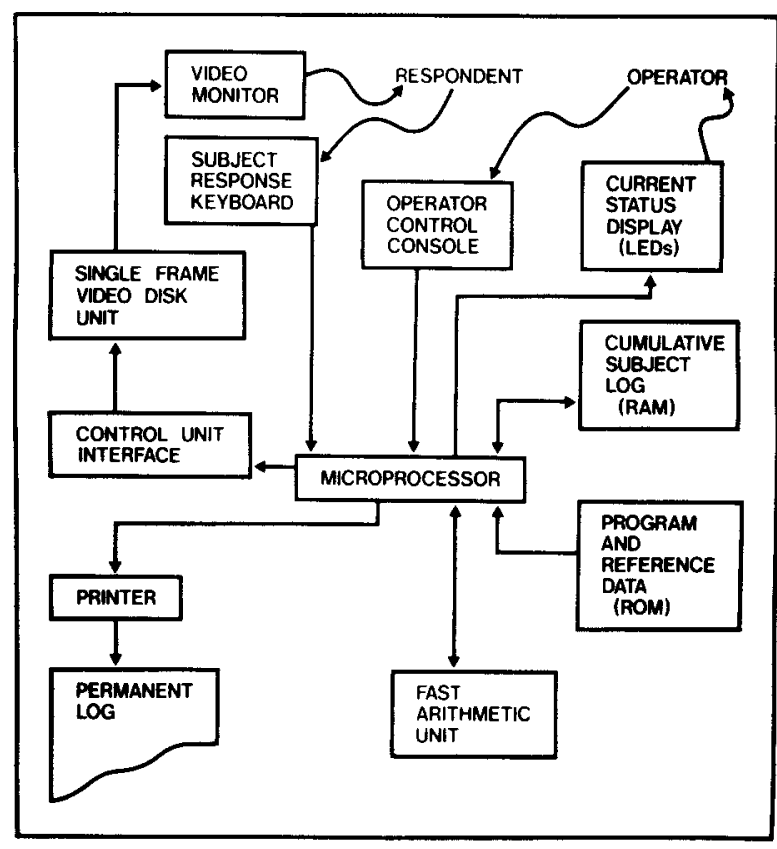

Figure 2. Diagram of the proposed Especially Rapid Diagnostic System (ERDS).

Twenty such categories would call for 20 vector elements and require 80 bytes. (3) The matrix of conditional probabilities $P\left(a_{j} / c_{i}\right)$ of a response to each item $j$ for members of a category i. Assuming a pool of 100 diagnostic items, this matrix would have $20 \times 100$ elements and require 8,000 bytes. (4) A critical value c, say, 0.99 .

This information may be stored in read-only memory (ROM) or on disk. If it is stored on disk, it must be read into read/write memory (random-access memory, RAM) prior to testing as part of the system initialization.

Each step of the procedure begins with a hypothesis. At Step 1, the hypothesis is determined by the base rates; at later steps, it is determined by the modified (i.e., posterior) probabilities $P\left(c_{i} / R\right)$, where $R$ is the vector of responses made by the patient. The posterior probabilities thus take into account the information obtained from the patient up to that point. For example, a patient might answer $b, a$, and $b$ to the first three items administered, yielding $R=\left(r_{1}, r_{2}, r_{3}\right)=\left(b_{1}, a_{2}, b_{3}\right)$, where $r$ is a response that could be either $a$ or $b$.

The updated or posterior probabilities of membership in the diagnostic categories are computed using the formula

$$
\begin{aligned}
P\left(c_{i} / R\right) & =P\left(c_{i} / r_{1}, r_{2}, \ldots r_{h}\right) \\
& =\frac{P\left(c_{i}\right) P\left(r_{1} / c_{i}\right) \ldots P\left(r_{h} / c_{i}\right)}{\sum_{k=1}^{g} P\left(c_{k}\right) P\left(r_{1} / c_{k}\right) \ldots P\left(r_{h} / c_{k}\right)},
\end{aligned}
$$

where $h$ is the number of responses up to and including Step $h$ and $g$ is the number of diagnostic categories.
These updated probabilities are stored in a separate matrix in RAM. Given a maximum of 100 items, this matrix has $20 \times 100$ elements and also requires 8,000 bytes. The computer also must store the item administered and the response made at each step. The total memory required in ROM and RAM may thus be around 32,000 bytes, and several commercially available microcomputer systems can serve as the center piece of ERDS.

The updated probabilities of membership in the various categories indicate at each step how the process is developing and whether it appears to be converging on a particular category. Access to them would allow the operator to estimate whether additional time is required and whether the patient should be detained or allowed to take a break. For this reason, the computer is connected to a display of light-emitting diodes (LEDs) on which the highest probabilities or similar information can be displayed.

When the testing is completed, either because the critical value $c$ has been attained or because all items have been administered, the operator can print the results obtained at each step to produce a permanent $\log$, as shown in Table 1 , before the information is erased during the testing of the next patient. Such logs would contain information potentially useful in evaluating the performance of the system, and they might provide information on the individual patients and/or the diagnostic categories.

\section{FUTURE POSSIBILITIES}

The system proposed here cannot handle a large number of diagnostic categories or diagnostic items. In spite of the rapid advances in microelectronics, the amount of information that can be manipulated using an on-site microcomputer system is limited, whereas a diagnostic system that does justice to the complexity of human nature would require a large number of diagnostic categories and items.

This raises a larger issue. A sophisticated and automated diagnostic system may be worth developing only if it can serve many users, that is, if it is a general classi-

Table 1

Hypothetical Log for a Patient Assuming Five Categories and 65 Administered Diagnostic Items

Patient Name: John Doe Date: August 18, 1980

Probability That Patient Belongs to Category

\begin{tabular}{crrrrrrr}
\cline { 3 - 7 } Step & \multicolumn{1}{c}{ Item Response } & $\mathbf{1}$ & 2 & 3 & 4 & 5 \\
\hline 1 & 1 & $\mathrm{~A}$ & .30 & .25 & .30 & .10 & .05 \\
2 & 25 & $\mathrm{~B}$ & .28 & .24 & .30 & .13 & .05 \\
3 & 43 & $\mathrm{~B}$ & .22 & .26 & .28 & .20 & .04 \\
. &. &. &. &. &. &. & $\cdot$ \\
. &. &. &. &. &. &. &. \\
. &. &. &. &. &. &. &. \\
65 & 12 & $\mathrm{~A}$ & .00 & .00 & .00 & .99 & .01 \\
\hline
\end{tabular}


fication system rather than merely a diagnostic system for psychiatric patients.

Let us assume for one optimistic moment that futuristic systems of classification, perhaps including a sequential Bayesian identification procedure and computer graphics, are worth considering further. A rather bold application of some of the ideas suggested here could involve a new information system called Telidon (e.g., Madden, 1979b), currently under development at the Canadian Department of Communications. Telidon involves a "smart" terminal attached to an ordinary television set. The modified set can be used to display textual and graphic material originating in a large and central computer data base. Given future development in computer graphics and the use of optical fibers with transmission capacities more than 100 times that of coaxial cables, such a system could become available to individual households for the purpose of classifying users into a variety of categories in terms of physical and mental health and in terms of educational and work requirements.

Users would sit in front of their NABUs, or "natural access bidirectional utilities" (a name whimsically suggested by Godfrey, 1979, for home computers of the future, which Madden, 1979a, even more tongue-incheek, labels AYEEs, for AEs, or alter egos). Responding to text or images, primarily the latter if society is moving toward more ideographic modes of expression, as Thompson (Note 1) suggests, users would determine whether they need medical or other professional help, in which educational program they should enroll, and for what available jobs they should apply. Their NABUs would, of course, supply the names and address codes of doctors, psychologists, hospitals, registrars of educational institutions, and manpower officials to be contacted, as well as a diagnostic statement that would reduce considerably the effort required to communicate their needs.

\section{REFERENCE NOTE}

1. Thompson, G. B. Memo from Mercury: Information technology is different (Occasional Paper No. 10). Montreal: Institute for Research on Public Policy, 1979.

\section{REFERENCES}

American Psychiatric Association. Diagnostic and statistical manual of mental disorders (3rd ed.). Washington, D.C: Author, 1980.

Bremser, R. F., \& Davidson, R. S. Microprocessor-assisted assessment in the clinical research laboratory. Behavior Research Methods \& Instrumentation, 1978, 10, 582-584.

Cowan, L., Auld, F., \& Begin, P. E. Evidence for distinctive personality traits in alcoholics. British Journal of Addiction, 1974, 69, 199-205.

Finney, J. C. Programmed interpretation of MMPI and CPI. Archives of General Psychiatry, 1966, 15, 75-82.

Fowler, R. D. The current status of computer interpretation of psychological tests. American Journal of Psychiatry, 1969, 125(Supplement), 21-27.

GoDFREY, D. Introduction: All information in all places at all times. In D. Godfrey \& D. Parkhill (Eds.), Gutenberg 2: The new electronics and social change. Toronto: Press Porcepic, 1979.

Gustafson, D. H. Evaluation of probabilistic information processing in medical decision making. Organizational Behavior and Human Performance, 1969, 4, 20-34.

Johnson, J. H., Giannetti, R. A., \& Williams, T. A. A selfcontained microcomputer system for psychological testing. Behavior Research Methods \& Instrumentation, 1978, 10, 579-581.

Johnson, J. H., \& Williams, T. A. Using a microcomputer for on-line psychiatric assessment. Behavior Research Methods \& Instrumentation, 1978, 10, 576-578.

Madden, J. Julia's dilemma. In D. Godfrey \& D. Parkhill (Eds.), Gutenberg 2: The new electronics and social change. Toronto: Press Porcepic, 1979. (a)

Madden, J. Simple notes on a complex future. In D. Godfrey \& D. Parkhill (Eds.), Gutenberg 2: The new electronics and social change. Toronto: Press Porcepic, 1979. (b)

Morf, M. E., \& Krane, W. R. A strategy for developing automated assessment systems. Behavioral Science, 1973, 18, 294-306.

Morf, M., Syrotuik, J., \& Krznaric, S. Real data simulation of a two-stage statistical diagnostic system. Journal of Consulting and Clinical Psychology, 1977, 45, 822-828.

Overall, J., \& Gorham, D. The brief psychiatric rating scale. Psychological Reports, 1962, 10, 799-812.

PaITich, D. A comprehensive automated psychological examination and report (CAPER). Behavioral Science, 1973, 18, 131-136.

RUDzINski, D. J. Development and validation of a picture. preference-test thought disorder scale. Unpublished doctoral dissertation, University of Windsor, 1979.

NOTE

1.485 E. Middlefield Road, Mt. View, California 94043. 